Reprod. Nutr. Dévelop., 1985, 25 (1 B), 235-241.

\title{
Lipoprotéines plasmatiques et engraissement : description d'un modèle chez le poulet domestique, Gallus domesticus
}

\author{
Dominique HERMIER, M. J. CHAPMAN (*)
}

Station de Recherches avicoles, I.N.R.A., Nouzilly 37380 Monnaie, France.

(*) I.N.S.E.R.M. U9, Pavillon Benjamin Delessert, Hopital de la Pitié, 83, bd de l'Hopital, 75013 Paris.

Summary. Plasma lipoproteins and fattening : description of a model in the domestic chicken, Gallus domesticus.

Developmental changes in the lipid transport system in two lines of broilers, selected for high or low adiposity, have been investigated during the first 7 weeks after hatching. The major lipoprotein classes in the plasma of lean and fat chickens 2, 3, 4, 5 and 7 weeks old were isolated by density gradient ultracentrifugation. The 2-week old chickens presented an heterogeneous distribution of high-density lipoproteins (HDL), often appearing as two distinct gradient bands; their very-low-density lipoproteins (VLDL) were rich in cholesterol $(25-30 \%)$ and relatively poor in triglycerides $(\sim 35 \%)$. As the birds grew older, the HDL subclass of lower density disappeared, tending to fuse with that of higher density; at the same time, the triglyceride content of VLDL and HDL levels increased progressively in the two lines, their concentration being significantly higher in the fat line. These developmental changes suggest that our two lines of chickens are a suitable model for studies of the onset and hormonal control of the specific fattening process during growth.

\section{Introduction.}

La production d'un poulet de chair à viande maigre, qui est devenue une nécessité zootechnique au cours de ces dernières années, passe par la compréhension des mécanismes qui contrôlent l'engraissement au cours de la croissance. C'est dans ce but qu'on été sélectionnées de façon divergente deux lignées de poulets de chair selon leur adiposité faible (lignée " maigre ») ou élevée (lignée " grasse " (Leclercq et al., 1980). Les différences apparues entre les deux lignées dans la régulation du métabolisme des lipides, en particulier l'hypoglycémie et I'hyperinsulinisme post-prandiaux de la lignée grasse (Touchburn et al., 1981), ainsi que l'accroissement de la lipogenèse hépatique dans la même lignée (Saadoun et Leclercq, 1983), laissent supposer des modifications quantitatives et/ou qualitatives des systèmes de transport des lipides. Le travail présenté ici résume les carac- 
téristiques des lipoprotéines dans les deux lignées, tout d'abord à l'âge de 5 semaines, choisi comme référence dans l'étude des paramètres biochimiques des deux lignées ; les modifications des différentes classes de lipoprotéines observées entre 2 et 7 semaines d'âge, permettent ensuite de préciser les modalités d'apparition des caractères biochimiques propres à chaque lignée dans les premiers stades du développement post embryonnaire.

\section{Matériels et méthodes.}

Animaux. - Seize poulets de la $6^{e}$ génération, 8 de la lignée maigre et 8 de la lignée grasse, âgés de 5 semaines, ont servi à la détermination du profil, de la concentration et de la composition chimique des différentes classes de lipoprotéines plasmatiques. Dans un deuxième temps, les variations de concentration plasmatique et de composition chimique de ces lipoprotéines en fonction de l'âge ont été étudiées à $2,3,4,5$ et 7 semaines sur un lot de 46 animaux de la $7^{\text {e }}$ génération.

Les animaux étaient élevés en cages individuelles et recevaient ad libitum un régime classique pour poulets de chair. Après un jeûne d'une nuit, le sang a été prélevé par voie-intracardiaque, recueilli sur EDTA et maintenu à $0{ }^{\circ} \mathrm{C}$. Le plasma a été séparé par centrifugation puis additionné d'EDTA, d'azide de sodium et de merthiolate de sodium à des concentrations finales de 0,$04 ; 0,01$ et $0,001 \%$ respectivement.

Séparation et caractérisation des lipoprotéines. - Pour chaque plasma, les différentes classes de lipoprotéines plasmatiques ont été séparées en une seule étape par ultracentrifugation en gradient de densité (Chapman et al., 1981). Les modifications apportées à cette technique, la détermination des limites de densité pour chaque classe, ainsi que les méthodes de dosage des protéines (PR), du cholestérol libre (CL), des esters du cholestérol (EC), des triglycérides (TG) et des phospholipides ( $P L)$, ont été décrites précédemment (Hermier et al., 1984). Nous avons considéré les classes de lipoprotéines plasmatiques suivantes : very low density lipoproteins (VLDL, de densité inférieure à $1,013 \mathrm{~g} / \mathrm{ml}$ ), intermediate density lipoproteins (IDL, de densité comprise entre 1,013 et $1,023 \mathrm{~g} / \mathrm{ml}$ ), low density lipoproteins ( $L D L, 1,023$ à $1,046 \mathrm{~g} / \mathrm{ml}$ ), et high density lipoproteins ( $\mathrm{HDL}, 1,052$ à $1,130 \mathrm{~g} / \mathrm{ml})$.

\section{Résultats.}

Caractéristiques des lipoprotéines à 5 semaines. - Chez les animaux à jeun de 5 semaines, la lignée grasse se caractérise par un taux plasmatique plus élevé d'esters du cholestérol, de triglycérides et de phospholipides.

Ces différences entre lignées se retrouvent au niveau des concentrations plasmatiques des lipoprotéines, surtout les VLDL, dont le taux est multiplié par deux chez les poulets gras (tabl. 1). Cette augmentation porte d'ailleurs sur tous les constituants de ces VLDL, y compris les protéines. 
TABLEAU 1

Concentrations plasmatiques des lipoprotéines chez les poulets maigres et gras de 5 semaines à jeun (en $\mathrm{mg} / \mathrm{ml}$ ).

\begin{tabular}{cccc}
\hline Lipoprotéines & $\begin{array}{c}\text { Maigres } \\
(8)\end{array}$ & $\begin{array}{c}\text { Gras } \\
(9)\end{array}$ & $\begin{array}{c}\text { Signification de } \\
\text { l'effet lignée }\end{array}$ \\
\hline VLDL & $0,03 \pm 0,01$ & $0,06 \pm 0,02$ & $\mathrm{P}<0,01$ \\
IDL & $0,28 \pm 0,08$ & $0,33 \pm 0,12$ & $\mathrm{P}<0,1$ \\
LDL & $1,09 \pm 0,20$ & $0,94 \pm 0,20$ & $\mathrm{NS}$ \\
HDL & $4,67 \pm 0,31$ & $5,16 \pm 0,47$ & $\mathrm{P}<0,05$ \\
\hline
\end{tabular}

Les valeurs sont des moyennes \pm écart-type du nombre d'échantillons indiqué entre parenthèses.

Les IDL montrent également un taux plus élevé chez les animaux gras, tandis qu'on n'observe aucune différence entre les taux de LDL plasmatiques des 2 lignées. Enfin, les HDL présentent un taux supérieur chez les animaux gras, avec une augmentation significative des quantités de phospholipides et de triglycérides dans cette classe.

Quant à la composition chimique des différentes classes de lipoprotéines, elle est identique dans les deux lignées; en particulier, le pourcentage de triglycérides n'est pas significativement différent (tabl. 2).

TABLEAU 2

Compositions chimiques des lipoprotéines plasmatiques chez les poulets maigres et gras de 5 semaines à jeun (en pourcentage des poids).

\begin{tabular}{cccccccc}
\hline $\begin{array}{c}\text { Lipopro- } \\
\text { téines }\end{array}$ & $\begin{array}{c}\text { Densité } \\
(\mathrm{g} / \mathrm{ml})\end{array}$ & $\mathrm{CL}$ & $\mathrm{CE}$ & $\mathrm{TG}$ & $\mathrm{PL}$ & $\mathrm{PR}$ & \\
\hline \multirow{2}{*}{ VLDL } & $<1,013$ & 3,1 & 15,0 & 41,7 & 15,2 & 26,8 & Maigres \\
& 3,9 & 14,9 & 44,6 & 14,1 & 22,4 & Gras \\
\hline \multirow{2}{*}{ IDL } & $1,013-$ & 7,3 & 30,3 & 14,4 & 17,7 & 29,8 & Maigres \\
& 1,023 & 6,7 & 26,6 & 18,8 & 19,1 & 28,6 & Gras \\
\hline \multirow{2}{*}{ LDL } & $1,023-$ & 10,1 & 30,4 & 7,5 & 21,9 & 29,7 & Maigres \\
& 1,046 & 10,8 & 29,9 & 7,5 & 22,3 & 29,5 & Gras \\
\hline \multirow{2}{*}{ HDL } & $1,052-$ & 3,9 & 23,4 & 1,7 & 28,6 & 45,4 & Maigres \\
& 1,130 & 3,6 & 21,8 & 1,6 & 28,1 & 44,9 & Gras \\
\hline
\end{tabular}

Evolution en fonction de l'âge. - L'observation des gradients de densité a permis de découvrir l'existence de deux classes de HDL chez certains des animaux les plus jeunes : 6 poulets sur 13 de 2 semaines, 3 sur 10 de 3 semaines et 1 sur 12 de 4 semaines, présentent une bande supplémentaire qui a comme limites de densité $1,045-1,080 \mathrm{~g} / \mathrm{ml}$. La bande normale des HDL se trouve ainsi décalée vers le bas : sa densité hydratée $(1,080-1,146 \mathrm{~g} / \mathrm{ml})$ est d'ailleurs supérieure à celle de la bande unique de HDL observée à 5 semaines $(1,052-1,130 \mathrm{~g} / \mathrm{ml}$ ) (tabl. 3 ).

L'évolution des taux plasmatiques des lipoprotéines chez les poulets à jeun est représentée sur la figure 1 . Jusqu'à 4 semaines, le taux de VLDL demeure à 
TABLEAU 3

Composition chimique des $H D L$ des poulets gras âgés de 2 et 5 semaines.

\begin{tabular}{ccccc}
\hline & \multicolumn{2}{c}{2 semaines } & & 2 semaines \\
2 bandes & & 1 bande & $\begin{array}{c}5 \text { semaines } \\
1 \text { bande }\end{array}$ \\
\cline { 2 - 5 } $\begin{array}{c}\text { Densité } \\
(\mathrm{g} / \mathrm{ml})\end{array}$ & $\begin{array}{c}\text { HDL-A } \\
1,045-1,080\end{array}$ & $\begin{array}{c}\text { HDL-B } \\
1,080-1,146\end{array}$ & $\begin{array}{c}\text { HDL } \\
1,050-1,146\end{array}$ & $\begin{array}{c}\text { HDL } \\
1,052-1,130\end{array}$ \\
\hline $\mathrm{CL}$ & 7,4 & 3,8 & 1,9 & 3,9 \\
EC & 26,1 & 19,9 & 21,1 & 23,4 \\
TG & 1,9 & 1,2 & 1,4 & 1,7 \\
PL & 26,6 & 22,9 & 24,2 & 28,6 \\
PR & 38,0 & 55,6 & 49,1 & 45,4 \\
\hline
\end{tabular}

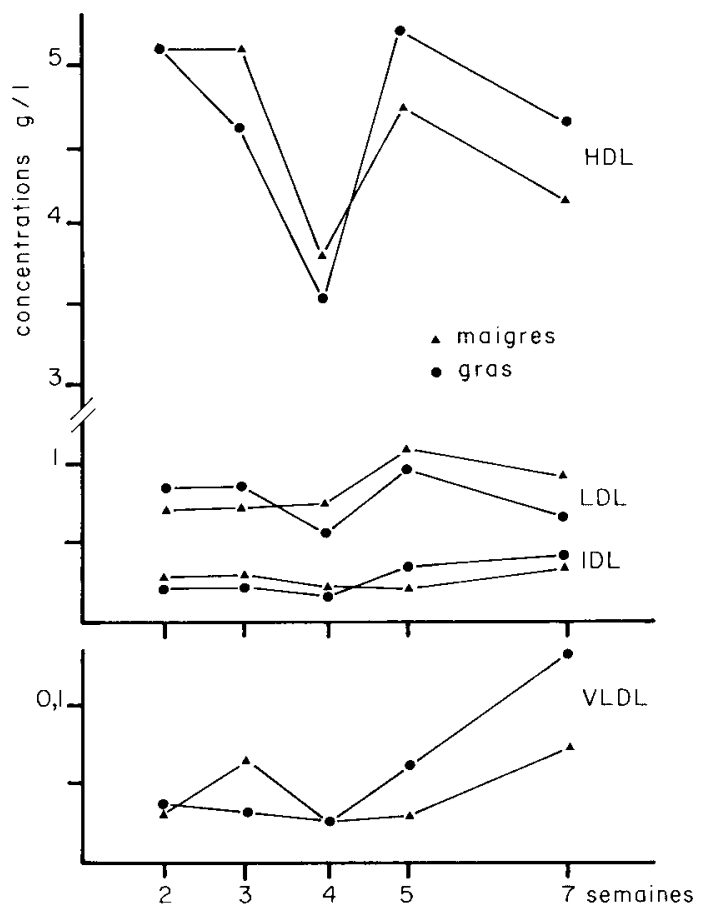

FIG. 1. - Evolution du taux plasmatique des lipoprotéines chez des poulets âgés de 2 à 7 semaines.

peu près constant, aux environs de $0,3 \mathrm{~g} / \mathrm{I}$ (sauf pour la lignée maigre qui devient à 3 semaines très supérieure à la lignée grasse). A partir de 4 semaines, ce taux augmente régulièrement, tandis que les différences entre les lignées s'accentuent avec l'âge au profit de la lignée grasse. Le taux de IDL évolue parallèlement à celui des VLDL avec une valeur minimum à 4 semaines et une différence significative entre les lignées à partir de cet âge. Le taux de LDL demeure à peu près constant, aux environs de $1 \mathrm{~g} / \mathrm{l}$, avec un maximum à l'âge de 5 semaines. Un taux plus faible à 4 et 7 semaines s'accompagne curieusement d'une différence 
significative entre les lignées au profit des animaux gras qui présentent alors une concentration supérieure de LDL. Le taux de HDL semble assez variable avec l'âge. Comme pour les VLDL et les LDL, il existe un minimum à l'âge de 4 semaines, stade à partir duquel les 2 lignées divergent significativement.

En ce qui concerne la composition chimique de ces classes de lipoprotéines, seules les VLDL présentent une évolution caractéristique (fig. 2) : dans les deux lignées, le pourcentage de triglycérides augmente jusqu'à 4 semaines tandis que celui du cholestérol diminue. Le pourcentage d'esters du cholestérol et des triglycérides, représentant le cœur de la particule de VLDL, demeure lui, sensiblement constant quel que soit l'âge considéré. Le pourcentage de triglycérides est significativement plus élevé dans la lignée grasse à l'âge de 3 semaines. Aux autres stades, on n'observe qu'une tendance dans le même sens. Considérées séparément, les deux bandes de HDL, dont les limites de densité encadrent celles de la bande classique à 5 semaines, ont aussi une composition chimique située de part et d'autre de celle des HDL à 5 semaines. Le tableau 3 compare les compositions

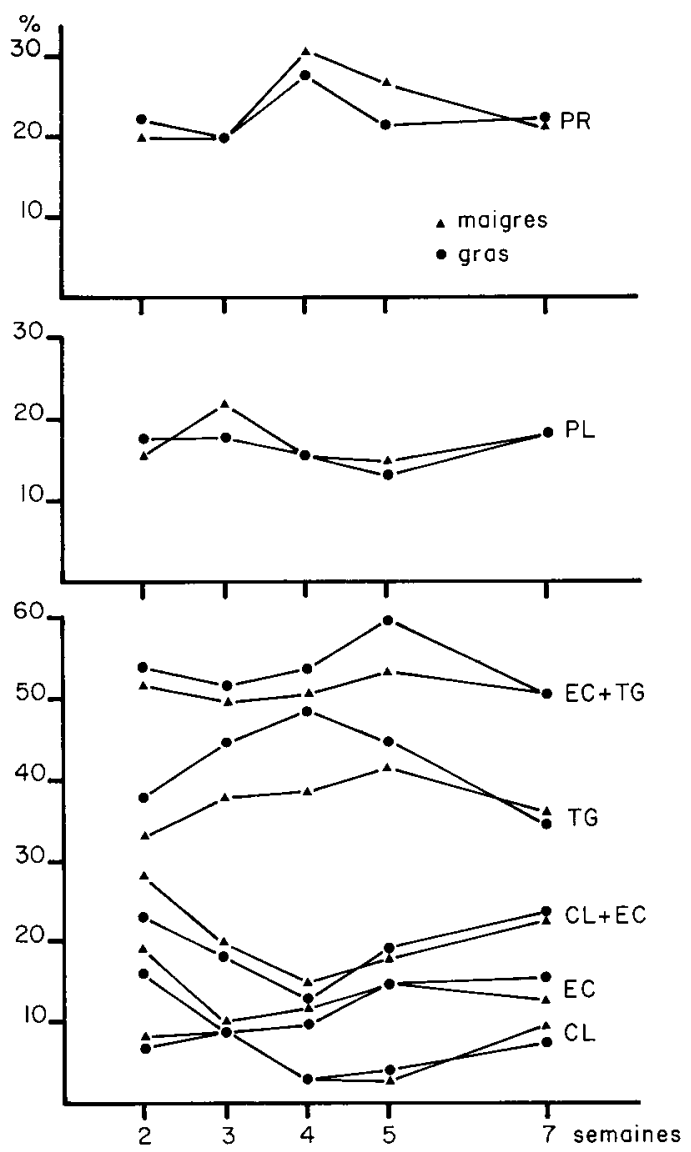

FIG. 2. - Composition chimique des VLDL de poulets maigres et gras âgés de 2 à 7 semaines. 
chimiques des HDL des animaux maigres de 2 semaines à celles des HDL à 5 semaines. La bande supplémentaire (HDL-A), de densité $1,045-1,080 \mathrm{~g} / \mathrm{ml}$ est plus riche en triglycérides et plus pauvre en protéines; bien que légèrement moins dense, elle se rapproche des HDL-2 humaines. La bande HDL-B, de densité $1,080-1,146 \mathrm{~g} / \mathrm{ml}$, est plus pauvre en triglycérides et plus riche en protéines ; bien qu'ayant une densité voisine de celle des HDL-2 humaines, elle est plus proche des HDL-3 par sa composition. Le rapport HDL-B/HDL-A est de l'ordre de 2.

\section{Discussion.}

La comparaison des systèmes de transport des lipides dans les deux lignées à jeun montre que la lignée grasse se caractérise par un taux de lipides plasmatiques plus élevé, particulièrement en ce qui concerne les triglycérides. Cette hyperlipidémie est le reflet des concentrations plus élevées des VLDL, des IDL qui en dérivent, et des HDL, qui pourraient résulter d'une intensification de la synthèse et de l'assemblage des particules de lipoprotéines par le foie. En effet, chez les oiseaux, cet organe est le principal responsable de la néosynthèse lipidique (Leveillé et al., 1975) ; I'on sait d'autre part que la synthèse hépatique des acides gras est plus élevée dans la lignée grasse (Saadoun et Leclercq, 1983). Il est intéressant de constater que l'augmentation des synthèses hépatiques concerne non seulement les triglycérides, mais aussi les autres lipides, et certaines apolipoprotéines, nécessaires à la structure et au métabolisme des particules de lipoprotéines.

En ce qui concerne l'évolution des lipoprotéines plasmatiques avec l'âge, le stade de 4 semaines semble décisif : la bande supplémentaire de HDL disparaît pour s'incorporer à la bande classique, les VLDL présentent un taux maximal de triglycérides et minimal de cholestérol, tandis que les concentrations plasmatiques des lipides et des lipoprotéines présentent une chute temporaire. On peut penser qu'on assiste à cet âge à une transition entre 2 types de métabolisme lipidique. En effet, les premières semaines de vie du poussin sont marquées par l'élimination du cholestérol stocké par le foie de l'embryon (Goy-Loeper et al., 1982), ce qui se traduirait par la diminution régulière du pourcentage de cholestérol dans les VLDL que nous avons observé dans les 2 lignées. Par ailleurs, on sait que, chez les oiseaux les VLDL contribuent à la formation des HDL (Behr et al., 1981). De la même façon, chez l'homme, la transformation des HDL3 en HDL2 est liée à un enrichissement des premières en fragments de surface provenant des lipoprotéines les plus riches en triglycérides et contenant en particulier du cholestérol libre (Patsch et al., 1978). On peut donc se demander si l'existence de deux classes de HDL chez le jeune poulet n'est pas liée elle aussi au métabolisme particulier du cholestérol associé à cet âge aux lipoprotéines riches en triglycérides. A partir de 4 semaines, les caractéristiques propres au métabolisme lipidique lié à la croissance et à l'engraissement peuvent se manifester pleinement, ce qui se traduit par une augmentation régulière du taux des VLDL plasmatiques, tandis qu'apparaissent entre les 2 lignées des différences qui ne font que s'accentuer avec l'âge. 
En conclusion, les deux lignées de poulets de chair sélectionnées sur leur adiposité faible ou élevée représentent un bon modèle pour l'étude des phénomènes de transport des lipides chez les oiseaux : absorption intestinale, sécrétion des lipides synthétisés de novo par le foie, captation et dégradation par le tissu adipeux. Les différences apparues entre les lignées sont à mettre en relation avec le déséquilibre glucose/insuline qui existe dans la lignée grasse et permettent d'aborder par ce biais les mécanismes de la régulation hormonale du métabolisme des lipides. Enfin, les liproprotéines représentent un bon marqueur dans l'étude des phénomènes d'engraissement lié à la croissance, en particulier dans tous les cas où l'on recherche des paramètres biochimiques susceptibles de variation génétique.

10. Réunion du groupe Développement I.N.R.A., Rennes, 9-10 mai 1984.

\section{Références}

BEHR S. F., PATSCH J. R., FORTE T., BENSADOUN A., 1981. Plasma lipoprotein changes resulting from immunologically blocked lipolysis. J. Lipid Res., 22, 443-451.

CHAPMAN M. J., GOLDSTEIN S., LAGRANGE D., LAPLAUD P. M., 1981. A density gradient ultracentrifugal procedure for the isolation of the major lipoprotein classes from human serum. J. Lipid Res., 22, 339-358.

GOY-LOEPER J., BARRE D., MAROUIE G., 1982. Spontaneous change in plasma lipid and lipoprotein patterns in the newly hatched chick. IRCS Med. Sci., 10, 458.

HERMIER D., CHAPMAN M. J., LECLERCO B., 1984. Plasma lipoprotein profile in fasted and refed chickens of two strains selected for high or low adiposity. J. Nutr., 114, 1112-1121.

LECLERCO 8., BOYER J. P., BLUM J. C., 1980. Selecting broilers for low or high abdominal fat : initial observations. Br. Poult. Sci., 21, 107-113.

LEVEILLÉ G. A., ROMSOS D. R., YEH Y. Y., O'HEA E., 1975. Lipid biosynthesis in the chick. A consideration of site of synthesis, influence of diet and possible regulating mechanisms. Poult. Sci, 54, 1075-1093.

PATSCH J. R., GOTTO A. M. Jr., OLIVECRONA T., EISENBERG S., 1978. Formation of high density lipoprotein-2-like particules during lipolysis of very low density lipoproteins in vitro. Proc. nat. Acad. Sci. U.S.A., 75, 4519-4523.

SAADOUN A., LECLERCQ B., 1983. Comparaison of in vivo fatty acid synthesis of the genetically lean and fat chickens. Comp. Biochem. Physiol., 75 B, 641-644.

TOUCHBURN S. P., SIMON J., LECLERCO B., 1981. Evidence of a glucose insuline imbalance and effect of dietary protein and energy level in chickens selected for high abdominal fat content. J. Nutr., 111, 325-335. 